\title{
Compact Bow-tie Antenna for the Detection of Multiple Tumors
}

\author{
Abdullah K. Alqallaf ${ }^{1}$, Rabie K. Dib ${ }^{2}$ \\ ${ }^{1}$ Department of Electrical Engineering, College of Engineering and Petroleum, Kuwait University \\ Kuwait \\ al.qallaf@ku.edu.kw \\ ${ }^{2}$ Department of Electronics Engineering, College of Technological Studies, Public Authority of Applied Education and \\ Training \\ Kuwait \\ rk.dib@paaet.edu.kw
}

\begin{abstract}
In the last two decades, microwave imaging methods for early detection of possible tumors have received ample amount of attentions. As a non-ionizing radiator, the microwave imaging has a definite advantage over X-ray mammography which is currently the main method of breast cancer diagnosis. This paper presents a focused synthetic radar imaging approach to detect the possible existing tumors and to clearly identify their sizes and locations to assist the physicians in cancer prognosis. Both analytical and simulation results are obtained for assumed tumors of different sizes and locations. This is done by modeling the breast tissues and the design of illuminating antenna. Finally, the proposed study includes the required design elements of the optimized antenna used for accurately locating the tumors within a minimal margin of error.
\end{abstract}

Keywords: Synthetic Radar Imaging, Multi-Tumors Detection, Bow-tie Antenna, Signal Processing Techniques.

\section{Introduction}

Breast cancer is a complicated disease with a variety of different causes. According to the International Agency for Research on Cancer (IARC), the Geneva Cancer Registry database, the European Network of Cancer Registries and recent studies, breast cancer incidence among women under the age of forty has been increased dramatically over years [27]. Although more patients are surviving due to early screening tests, breast cancer is the most common cancer worldwide and it has been on the rise for several years. Early screening tests and examinations for breast cancer are vital to find the disease before it starts to cause symptoms.

The X-ray mammography has been proposed as the most approved method of breast imaging. However, it has basic limitations due to the small contrast between the diseased and normal tissue at the X-ray frequencies [2-5]. During imaging, the breast is compressed in order to create uniformity, otherwise heterogeneous tissue, and to avoid image blurring. This compression causes pain and at least inconvenience that may reduce the patient compliance to safety recommendations. Microwave detection of cancerous tumors has been of great interest as an alternative or a complementary method to ionizing radiation methods such as computed tomography or mammography. Furthermore, microwave imaging enjoys the advantage of a clear contrast between the healthy tissues and diseased tissues or tumors [6-9]. Recently, growing efforts appear in the literature for verifying the possibility of microwave imaging of the breast. Two approaches can be identified; the tomographic method that involves inverse scattering technique [11] and the confocal imaging that is based on the concept of synthetic radar imaging [17-20]. The tomographic method uses the backscattered waves in an algorithm to reconstruct an image of the breast, in terms of tissue electrical parameters. The problem is considered an ill-conditioned nonlinear one and normally requires intensive computational work [10-14]. This is so due to the heterogeneous nature of the breast medium. However some initial successful experimental results appear in [15-16]. The method of confocal microwave imaging is based on the concept of synthetic radar imaging where the antenna focuses waves on small volume within the breast. This volume is scanned to provide an image of the whole breast volume as studied by [17-20]. Further studies by [21-24] confirm the method by simulation and experiment. They also show the robustness of the method to variations of the dielectric properties of the breast tissues. Such variations cause clutter in the received response that can be reduced by the power of focusing. 
An attempt to further reduction of such clutter is described recently by [25] who uses a rotating antenna array. Two positions for the breast are used during imaging: the supine position and the prone position [20-24]. In the supine position the breast is oriented upwards and flattened out. A broadband antenna is placed on top and is fed by a suitable pulse. The return pulse is recorded and the early time response is cancelled since it comes from the skin layer. The response is taken at different antenna positions. Signal processing is then used, as in synthetic aperture radar for constructing an image. In the prone position, on the other hand, the breast is oriented downwards and therefore it resembles a cylinder. Again an antenna is placed on the cylindrical surface to send a broadband pulse. The return pulses for different antenna positions are used to image the breast. Figure 1 is a graphical representation of the human breast structure, antenna orientations and the breast tumors with different sizes and locations.

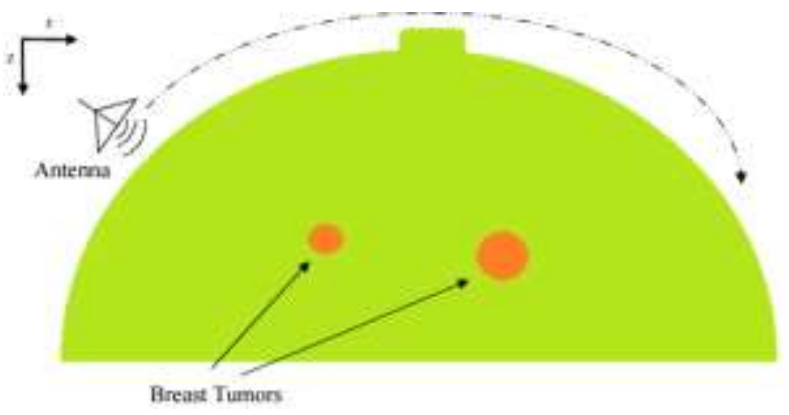

Fig. 1: Illustration of the human breast structure. The dashed curve represents the designed antenna path and the orange dots represent the breast tumors.

In this paper, we apply a focused synthetic radar imaging approach for the detection of the possible existing tumors. This is to assist the physicians in cancer prognosis to clearly map and detect the tumors locations and their sizes. The compact antenna used here is based on optimizing our previously designed antenna [1]. It shows that the optimum frequencies for detecting tumors depend on the tumors' radii and it is recommended that images should be taken at few distinct frequencies in the range $5-7 \mathrm{GHz}$ in order to detect tumors of different sizes.

The paper is organized as follows. Section 2 introduces the optimized antenna design structure and breast modeling. Section 3 presents the obtained simulation results and discussions. Finally, conclusion remarks based on the simulation results is presented in section 4 .

\section{Optimized Antenna Design and Experimental Modeling}

In general, there are two possible positions for the breast that can be used during the imaging process, the supine position and the prone position. In the supine position the breast is oriented upwards and flattened out, while in the prone position the breast is oriented downwards [17]. Here, we consider breast imaging in the supine position. The breast is assumed as a homogeneous medium having complex dielectric constant with conductivity $\sigma(\mathrm{S} / \mathrm{m})$ and relative permittivity $\varepsilon_{\mathrm{r}}$. The possible existing tumors are modeled as small spherical like shape inhomogeneity having contrast conductivity $\sigma_{\mathrm{s}}$ and relative permittivity $\varepsilon_{\text {rs. }}$ The breast is then modeled as a rectangular box with upper surface coinciding with $Z=0$ plane with a width of to $200 \mathrm{~mm}$ and a depth of $150 \mathrm{~mm}$. Possible tumors of spherical shape with different radii exist at a depth $d$, i.e, at $Z=-d$. Using Computer Simulation Technology (CST) Microwave Studio Suite, an antenna is placed with its phase center at the origin of $(x, y, z)$ coordinate system and radiates in the breast medium. While the relative permittivity $\varepsilon_{\mathrm{r}}$ of normal tissues is around 10, that of the tumor is 50 . There is also contrast between the conductivities, being about $0.15 \mathrm{~S} / \mathrm{m}$ for normal tissue and $0.7 \mathrm{~S} / \mathrm{m}$ for tumors [17]. Table 1 summarizes the conductivity and relative permittivity parameters of the human. The choice of a suitable antenna for breast cancer detection is very crucial matter. The antenna is required to be compact, wide band, low profile, lightweight, and flexible enough to be placed directly on the breast.

Table 1: summarization of the human tissues parameters of the breast and tumors [17].

\begin{tabular}{|c|c|c|}
\hline Tissue & Conductivity $\boldsymbol{\sigma}(\mathbf{S} / \mathbf{m})$ & Relative permittivity $\boldsymbol{\varepsilon}_{\mathbf{r}}$ \\
\hline Tumor & 0.7 & 50 \\
\hline Breast & 0.15 & 10 \\
\hline
\end{tabular}


Several antenna designs have been proposed for breast tumor detection including the dielectric resonator antenna [2223], the stacked patch antenna [24], the wide slot UWB antenna [25], and the flexible bowtie [26]. All of these antennas meet the required traits of a breast tumor sensor. Table 1 illustrates the conductivity and relative permittivity parameters of the human breast healthy tissues and the tumors as described in [17]. For this, a compact flexible bowtie antenna is used in this study as a detective probes handled by the operator. The optimized antenna geometry and dimensions are chosen as in Figure 2 and Table 2 to operate around the center frequency of $5.5 \mathrm{GHz}$. This frequency is chosen as a compromise between required depth of penetration in the tissues and the existing tumors locations with suitable resolution as described in [1]. The metal body of the bow-tie is printed on two thin flexible dielectric sheets as suggested by [26] and is fed by a microstrip line as shown in Figure 2. The total thickness of the two dielectric sheets equals $0.1287 \mathrm{~mm}$. The dielectric sheet is $0.05 \mathrm{~mm}$ thick and has a relative permittivity of 4 . A matching scheme is designed with the feed line as shown in Figure 2. The resulting reflection loss $\left|S_{11}\right|$, when the antenna is placed on a medium that resembles the breast electrical properties is given in Figure 3 . It is seen that a $-10 \mathrm{~dB}$ reflection loss or more is secured in the frequency ranges 5.2-6 GHz.

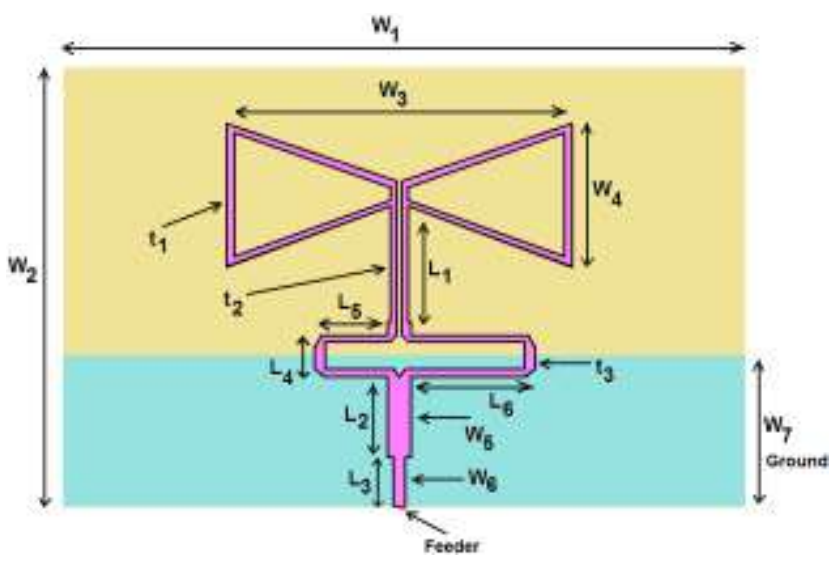

Fig. 2: Compact bow-tie antenna used for imaging. The optimized antenna dimensions are given in Table 2 . The antenna is placed on a medium with the breast-like permittivity.

Table 2: Compact bow-tie antenna dimensions used for imaging.

\begin{tabular}{|c|c|}
\hline Parameters & Length in (mm) \\
\hline $\mathrm{W}_{1}$ & 30 \\
\hline $\mathrm{W}_{2}$ & 20 \\
\hline $\mathrm{W}_{3}$ & 15.6 \\
\hline $\mathrm{W}_{4}$ & 6.34 \\
\hline $\mathrm{W}_{5}$ & 0.48 \\
\hline $\mathrm{W}_{6}$ & 0.37 \\
\hline $\mathrm{W}_{7}$ & 6 \\
\hline $\mathrm{L}_{1}$ & 6.45 \\
\hline $\mathrm{L}_{2}$ & 3.44 \\
\hline $\mathrm{L}_{3}$ & 1.48 \\
\hline $\mathrm{L}_{4}$ & 1.18 \\
\hline $\mathrm{L}_{5}$ & 2.14 \\
\hline $\mathrm{L}_{6}$ & 5.92 \\
\hline $\mathrm{t}_{1}$ & 0.3 \\
\hline $\mathrm{t}_{2}$ & 0.28 \\
\hline $\mathrm{t}_{3}$ & 0.28 \\
\hline
\end{tabular}




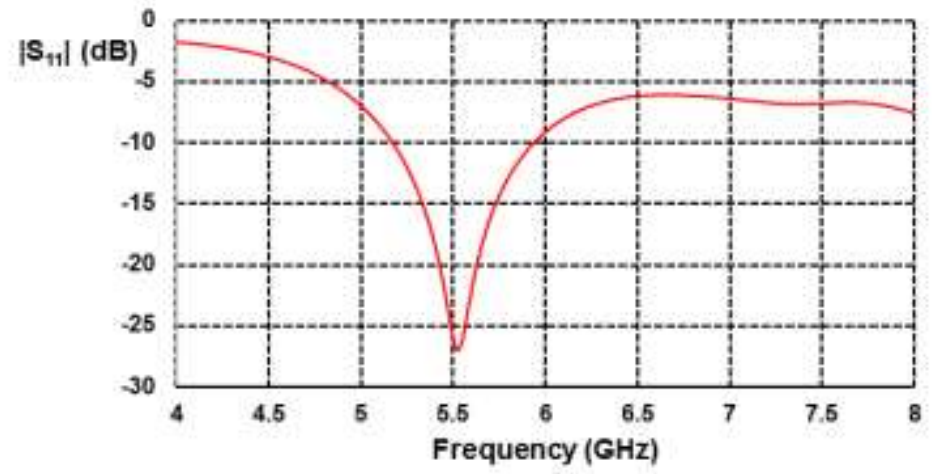

Fig. 3: The reflection of the compact bow-tie antenna $\left|\mathrm{S}_{11}\right|$ in $\mathrm{dB}, y$-axis, versus the frequency, in $\mathrm{GHz}, x$-axis.

\subsection{Theoretical Analysis}

To determine the scattered field from the inhomogeneity of the possible tumors, we assume that the diameters of the tumors are less than the applied wavelength. The incident wave is a single-plane wave focused from the antenna phase center at each position to the tumors centers in the radial coordinate $r$. With this assumption, the incident radial field can be determined from the sphere center in the form:

$$
E_{r}^{i}=\frac{E_{o} \cos \phi}{j k r} \sum_{n=0}^{\infty} j^{-n}(2 n+1) \hat{\jmath}_{n}(k r) \frac{\partial P_{n}^{(1)}(\cos \theta)}{\partial \theta}
$$

where $k$ is the complex wave number and $\hat{\jmath}_{n}(k r)$ is the spherical Bessel function and $P_{n}^{(1)}(\cos \theta)$ is the associated Legendre polynomial. The incident radial field in Eq. (1) is also derivable from a magnetic vector potential $A_{r}$, so that:

$$
E_{r}^{i}=(j \omega \epsilon)^{-1}\left[\frac{\partial^{2}}{\partial r^{2}}+k^{2}\right] A_{r}^{i}=(j \omega \epsilon)^{-1} \frac{n(n+1)}{r^{2}} A_{r}^{i}
$$

Therefore:

$$
A_{r}^{i}=\frac{E_{o} \cos \phi}{\omega \mu} \sum_{n=0}^{\infty} a_{n} J_{n}(k r) P_{n}^{(1)}(\cos \theta)
$$

where $a_{n}=j^{-n}(2 n+1) /(n(n+1))$. Similarly, for the radial magnetic field, the incident electric vector potential:

$$
F_{r}^{i}=\frac{E_{o} \sin \phi}{k} \sum_{n=0}^{\infty} a_{n} J_{n}(k r) P_{n}^{(1)}(\cos \theta)
$$

The scattered fields have similar form except that $\hat{\jmath}_{n}(k r)$ is replaced by the spherical Hankel function $\widehat{H}_{n}(k r)$. The total external field to the sphere is the sum of the incident and scattered fields, formally:

$$
\begin{aligned}
& A_{r}^{e x}=\frac{E_{o} \cos \phi}{\omega \mu} \sum_{n=0}^{\infty}\left[a_{n} J_{n}(k r)+b_{n} \widehat{H}_{n}(k r)\right] P_{n}^{(1)}(\cos \theta) \\
& F_{r}^{e x}=\frac{E_{o} \sin \phi}{k} \sum_{n=0}^{\infty}\left[a_{n} J_{n}(k r)+c_{n} \widehat{H}_{n}(k r)\right] P_{n}^{(1)}(\cos \theta)
\end{aligned}
$$


where $b_{n}$ and $c_{n}$ are the scattered field coefficients. The internal fields inside the spherical tumor, namely:

$$
\begin{aligned}
A_{r}^{i n} & =\frac{E_{o} \cos \phi}{\omega \mu} \sum_{n=0}^{\infty} d_{n} J_{n}(k r) P_{n}^{(1)}(\cos \theta) \\
F_{r}^{i n} & =\frac{E_{o} \sin \phi}{k_{s}} \sum_{n=0}^{\infty} e_{n} J_{n}(k r) P_{n}^{(1)}(\cos \theta)
\end{aligned}
$$

where again $d_{n}$ and $e_{n}$ are the internal fields coefficients and $k_{s}$ is the wave number in the spherical tumor. Applying the boundary conditions to determine the unknown coefficients $b_{n}$ and $c_{n}$ require the continuity of the $\theta$ and $\phi$-field components at the spherical surface $r=a$. The scattered field external region component is:

$$
E_{\theta}^{S}(r, \theta)=\frac{-E_{o}}{k r} \cos \phi \sum_{n=1}^{N} c_{n} \widehat{H}_{n}(k r) \frac{P_{n}^{\prime}(\cos \theta)}{\sin \theta}+j b_{n} \widehat{H}_{n}^{\prime}(k r) \frac{\partial P_{n}^{\prime}(\cos \theta)}{\partial \theta}
$$

here the prime on top of the Bessel and the Hankel spherical functions represents the differentiation with respect to the argument, $\underline{\eta}_{s}=\eta_{s} / \eta, \underline{k_{s}}=\frac{k_{s}}{k}$, and $R_{n s}=\widehat{J_{n}^{\prime}}\left(k_{s} a\right) / \widehat{H_{n}^{\prime}}\left(k_{s} a\right)$, where $\eta$ is the wave impedance in the breast medium. The summation term is truncated after $N$ terms depending on the tumor size. Arithmetic calculations show that $N=5$ gives acceptable convergence up to tumor radius of $7 \mathrm{~mm}$ at $5.5 \mathrm{GHz}$ applied frequency. The scattered field at the transmission is equal to: $E_{\theta}\left(r_{1}, \pi\right)\left(-\frac{d}{r_{1}}\right)$, where $r_{1}=\sqrt{d^{2}+L^{2}}$. Here, $d$ and $L$ are the vertical and the horizontal displacement between the focused antenna and the center of the tumor, respectively. The next step is to realize focusing on the tumor for $M$ locations of the antenna at $L=i \Delta ; i=0,1,2 \ldots(M-1)$. The total sum of the signals for the $M$ locations of the antenna is:

$$
E_{\text {total }}=\sum_{i=0}^{M-1}\left[E_{\theta}\left(r_{1}, \pi\right)\left(-\frac{d}{r_{1}}\right)\right] \exp \left[+2 j k r_{1}\right]
$$

The focused antenna is placed in 17 equi-spaced locations with an increment of $\Delta=10 \mathrm{~mm}$. The field is normalized relative to the incident radial field, $E$. The response tends to increase with the tumor radius, but in an oscillatory manner as noted in [1]. It was also shown that the theoretical and simulation results are in good agreements.

\subsection{Experimental Setup}

A practical model to the reality has been developed for simulating the breast tissue and a parametric study has been performed in the presence of multiple tumors in the tissue in which the trajectory of movement of the focused antenna. The designed antenna has be optimized to minimize the operational error in detecting every the tumors based on the finite element analysis of the scattering of the incident wave radiated by a wideband dipole antenna and received by itself positioned the way that satisfies the requirements in the absence of obstacle which is a tumor of any size. As described in the previous section, the critical dimensions of the wideband antenna design have been studied parametrically within the finite element simulations to optimize the contrast of the scattering among the cases of different number of presented tumors. The optimized antenna will be the detective probes handled by the operator in the final fabrication stage of the design. The full threedimensional solver for discrete number of frequency points has been employed to provide the radiation pattern variations in the design process. This is accurately deliver 3D electromagnetic simulations of the frequency using High Frequency Structure Simulator (HFSS), Computer Simulation Technology (CST) Microwave Studio and MATLAB programming. Figure 4 illustrates the general planned process of the experimental setup from the incident field until the image formation of tumors detection. 


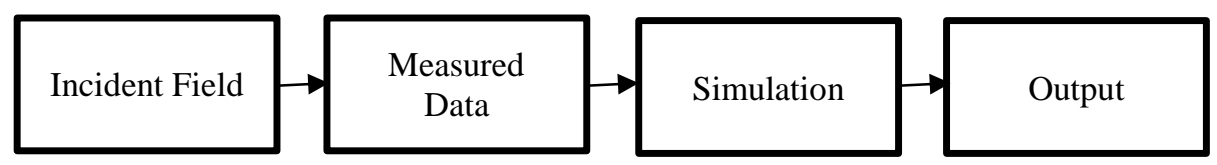

Fig. 4: General proposed process representation.

\subsection{Image Reconstruction Algorithm and Evaluation}

Since the scattered signal is measured in frequency-domain, hence, a comprehensive signal processing algorithm, namely, the Inverse Fast Fourier Transform (IFFT) has been used to transform the signal to time-domain. The time-domain backscatter signals are analyzed and an artifact removal is being applied using data adaptive algorithm to remove (subtract) the early time artifacts at the received signals of tumors reflection. This is for clearly mapping and locating of the detected tumors and to generate the exact coordinates to be used in the radiotherapy treatments. Figure 5 shows the process of image formation process. The measured data represent the scattered signal received from any position under consideration and the area with tumors and without tumors are subtractions in order to obtain the tumor. Then, the total sum imaging is applied with the consideration of time delay to get the spatial focusing of the backscattered signals for image formation of tumors location.

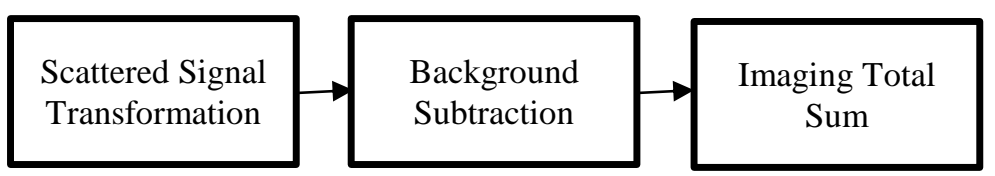

Fig. 5: Block diagram of the breast imaging reconstruction.

\section{Simulation Results and Discussions}

The antenna is placed on the breast surface and is fed by a pulse that covers a bandwidth of $500 \mathrm{MHz}$ around $5.5 \mathrm{GHz}$ center frequency. The pulse bandwidth is less than $10 \%$ of the center frequency. Within this narrow bandwidth, the breast electrical parameters can be assumed constants and therefore, one can accurately compensate for the propagation effects in the breast. One can apply the pulse at different discrete center frequencies to obtain multi-frequency images for the tumor. The voltage waveform picked up by the antenna is monitored for, say, $M$ different positions of the antenna as it is displaced along the $X$-axis. To remove the primary pulse and possible reflections from the skin layer of the breast, the average received waveform from the $M$ positions is subtracted from each of the received waveforms. The resulting signals represent the scattered field from the tumor inhomogeneity at the $M$ antenna positions. The next step is to process the $M$ signals so as to synthetically focus the field at an arbitrary point within the breast. This leads to imaging the breast medium. In processing the $M$ waveforms, we have to account for the variations of the antenna radiation pattern at the scanned points. The response obtained from a spherical tumor of radius $6 \mathrm{~mm}$ in the $X-Z$ plane is shown in Figure 6.

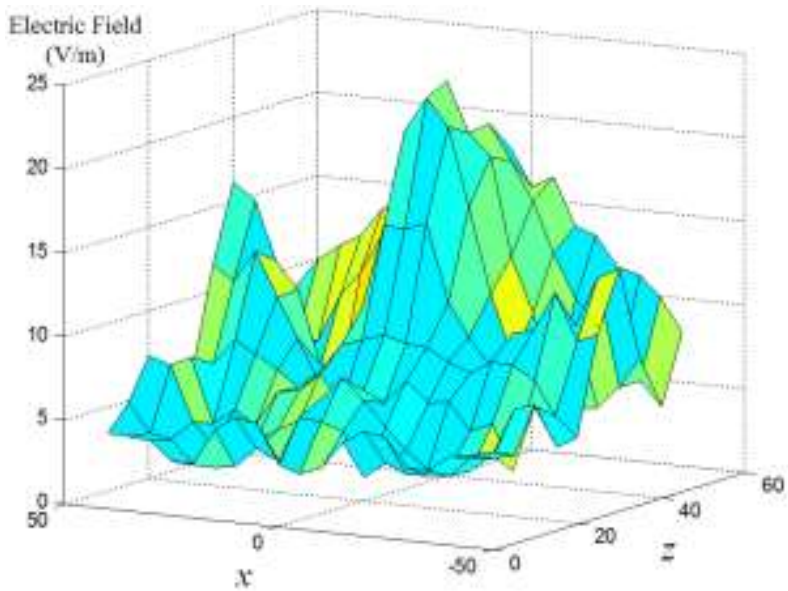

(a)

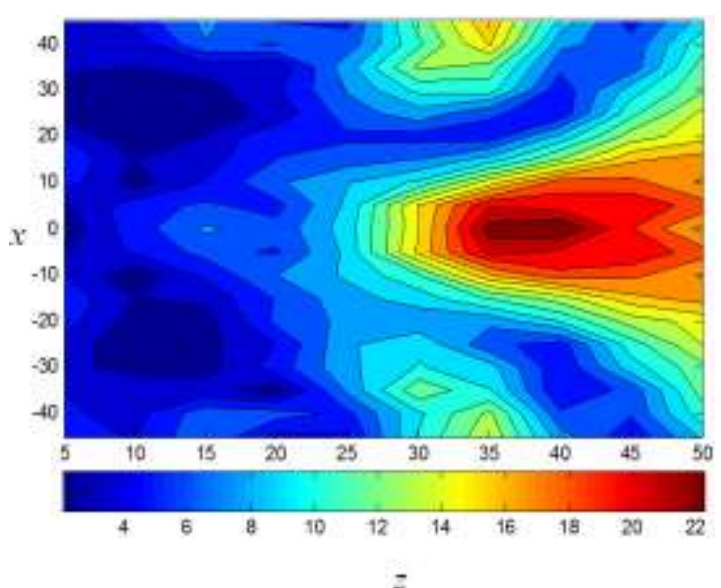

(b)

Fig. 6: Reflection response versus observing point on the $x-z$ plane for $6 \mathrm{~mm}$ radius tumor. a) 3-dimensional plot. b) Contours of constant reflection. 
The breast is modeled as a block of width $200 \mathrm{~mm}$, and depth of $100 \mathrm{~mm}$. The response in Figure 6a is obtained for $M=17$ positions of the antenna on the $Z=0$ plane. The contours of constant response levels are shown in Figure $6 \mathrm{~b}$. The obtained results in Figure 6 shows an agreement between the peak and tumor location with few millimeters shift Figure 7a,b show the same response as in Figure 6a,b, except that multi-tumors are suggested. It is clear from both figures that there is a clear peak response at the exact position and a little shifted depth of the tumor. The resolution may be determined by the region where the response is reduced to $50 \%$ of the peak value. For the single tumor of $6 \mathrm{~mm}$, the resolution region covers $20 \mathrm{~mm}$ about the $X$ position and about $15 \mathrm{~mm}$ in the $Z$-direction. This means that two tumors separated horizontally by less than $20 \mathrm{~mm}$ or vertically by $15 \mathrm{~mm}$ cannot be distinguished and will show as one tumor. It is interesting to study the reflection level as a function of the tumor radius at a given depth.

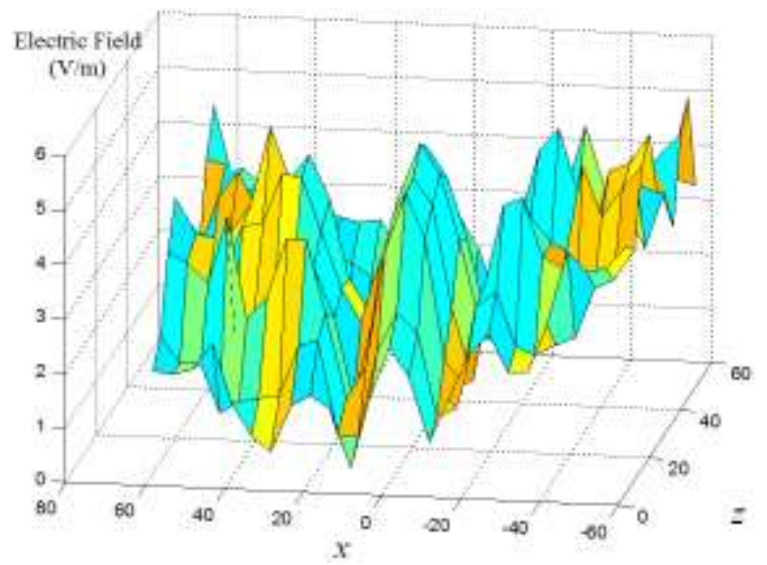

(a)

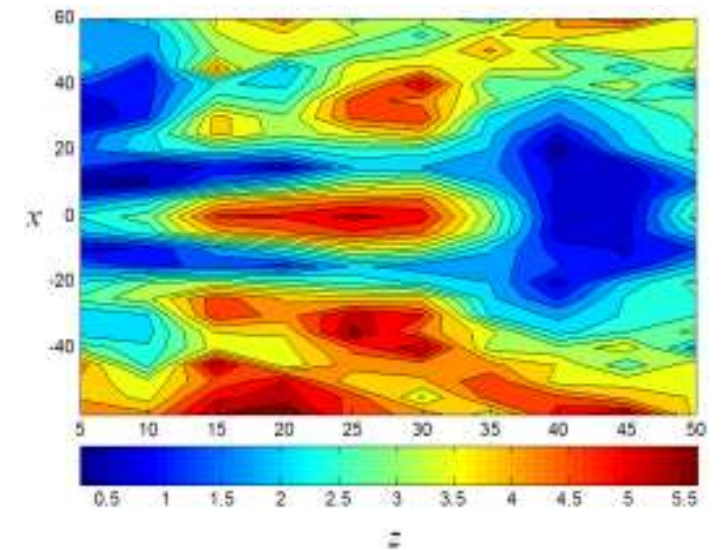

(b)

Fig. 7: Reflection response versus observing point on the $x$ - $z$ plane for $6 \mathrm{~mm}$ radii tumors. a) 3 -dimensional plot. b) Contours of constant reflection.

\section{Conclusion}

In this paper, we present a focused synthetic radar imaging approach for breast cancer screening using an optimized antenna. The proposed method provides a low-risk tool, non-ionized radiation, for the detection of multiple tumors simultaneously. This is done by modeling the breast as a homogeneous medium, while the tumors are modeled as small spherical inhomogeneity. To clearly map and locate the possible existing tumors, proposed approach is applied using compact antenna. It is demonstrated that the spherical-like tumor can be detected with reasonable resolution using a narrow band pulse of $5.5 \mathrm{GHz}$ center frequency and $0.5 \mathrm{GHZ}$ bandwidth. The simulation results are supported by analytical results where a reasonably good agreement is observed. Finally, the simulation results provide an evidence of detecting the possible existing tumors as small as small $(<4 \mathrm{~mm})$ at early stages with a minimal marginal error of a radius of at most $0.5 \mathrm{~cm}$ from their original locations within the breast phantom.

\section{Acknowledgements}

This work was supported by Kuwait University Research Grant No. [EE03/16].

\section{References}

[1] A. K. Alqallaf, R. K. Dib, S. F. Mahmoud, "Microwave Imaging Using Synthetic Radar Scheme Processing for the Detection of Breast Tumors," The Applied Computational Electromagnetics Society (ACES), vol. 31, no. 2, p. 98105 , 2016.

[2] M. Brown, F. Houn, E. Sickles, and L. Kessler, "Screening mammography in community practice," Amer. J. Roentgen., vol. 165, pp. 1373-1377, 1995.

[3] P. T. Huynh, A. M. Jarolimek, S. Daye, "The false-negative mammogram," Radiograph., vol. 18, no. 5, pp. 11371154, 1998.

[4] J. G. Elmore, M. B. Barton, V. M. Moceri, S. Polk, P. J. Arena, S. W. Fletcher, "Ten-year risk of false positive screening mammograms and clinical breast examinations," New Eng. J. Med., vol. 338, no. 16, pp.1089-1096, 1998. 
[5] V. P. Jackson, R. E. Hendrick, S. A. Feig, and D. B. Kopans, "Imaging of the radiographically dense breast," Radiology, vol. 188, pp. 297-301, 1993.

[6] A. J. Surowiec, S. S. Stuchly, J. R. Barr, and A. Swarup, "Dielectric properties of breast carcinoma and the surrounding tissues," IEEE Trans. Biomed. Eng., vol. BME-35, pp. 257-263, 1988.

[7] W. T. Joines, Y. Zhang, C. Li, and R. L. Jirtle, "The measured electrical properties of normal and malignant human tissues from 50 to $900 \mathrm{MHz}$," Med. Phys., vol. 21, pp. 547-550, 1994.

[8] S. S. Chaudhary, R. K. Mishra, A. Swarup, J. M. Thomas, "Dielectric properties of normal and malignant human breast tissues at radiowave and microwave frequencies," Indian J. Biochem. Biophys., vol. 21, pp. 76-79, 1984.

[9] S. C. Hagness, K. M. Leininger, J. H. Booske, M. Okoniewski, "Dielectric characterization of human breast tissue at microwave frequencies," $2^{\text {nd }}$ World Congr. Microwave and Radio Frequency Processing, Orlando, FL, Apr. 2000.

[10] S. Caorsi, G. L. Gragnani, and M. P. Pastorino, "Reconstruction of dielectric permittivity distributions in arbitrary 2D inhomogeneous biological bodies by a multiview microwave numerical method," IEEE Trans. Med. Imag., vol. 12, pp. 232-239, 1993.

[11] A. E. Souvorov, A. E. Bulyshev, S. Y. Semenov, R. H. Svenson, A. G. Nazarov, Y. E. Sizov, and G. P. Tatsis, "Microwave tomography: A two-dimensional Newton iterative scheme," IEEE Trans. Microwave Theory Tech., vol. 46, pp. 1654-1659, 1998.

[12] W. C. Chew, Advances in Computational Electrodynamics: The Finite-Difference Time-Domain Method, A. Taflove, ed. Norwood, MA: Artech House, 1998, ch. 12.

[13] A. Franchois, A. Joisel, C. Pichot, and J. C. Bolomey, "Quantitative microwave imaging with a 2.45-GHz planar microwave camera," IEEE Trans. Med. Imag., vol. 17, pp. 550-561, 1998.

[14] M. Bertero, M. Miyakawa, P. Boccacci, F. Conte, K. Orikasa, and M. Furutani, "Image restoration in chirp-pulse microwave CT (CP-MCT)," IEEE Trans. Biomed. Eng., vol. 47, pp. 690-699, 2000.

[15] P. M. Meaney, K. D. Paulsen, J. T. Chang, M. W. Fanning, A. Hartov, "Nonactive antenna compensation for fixedarray microwave imaging-Part II: Imaging results," IEEE Trans. Med. Imag., vol. 18, pp. 508-518, 1999.

[16] P. M. Meaney, M. W. Fanning, D. Li, S. P. Poplack, and K. D. Paulsen, "A clinical prototype for active microwave imaging of the breast," IEEE Trans. Microwave Theory Tech., vol. 48, pp. 1841-1853, 2000.

[17] S. C. Hagness, A. Taflove, and J. E. Bridges, "Two-dimensional FDTD analysis of a pulsed microwave confocal system for breast cancer detection: fixed-focus and antenna-array sensors," IEEE Trans. Biomed. Eng., vol. 45, pp. 1470-1479, 1998.

[18] S. C. Hagness, A. Taflove, and J. E. Bridges, "Wideband ultra low reverberation antenna for biological sensing," Electron. Lett., vol. 33, no. 19, pp. 1594-1595, 1997.

[19] S. C. Hagness, A. Taflove, and J. E. Bridges, "Three-dimensional FDTD analysis of a pulsed microwave confocal system for breast cancer detection: design of an antenna-array element," IEEE Trans. Antennas Propagat., vol. 47, pp. 783-791, 1999.

[20] X. Li and S. C. Hagness, "A confocal microwave imaging algorithm for breast cancer detection," IEEE Microwave Wireless Comp. Lett., vol. 11, pp. 130-132, 2001.

[21] E. Fear and M. Stuchly, "Microwave system for breast tumor detection," IEEE Microwave Guided Wave Lett., vol. 9, pp. 470-472, 1999.

[22] E. C. Fear and M. A. Stuchly, "Microwave detection of breast cancer," IEEE Trans. Microwave Theory Tech., vol. 48, pp. 1854-1863, 2000.

[23] E. C. Fear and M. A. Stuchly, "Microwave detection of breast tumors: Comparison of skin subtraction algorithms," Proc. SPIE, vol. 4129, pp. 207-217, 2000.

[24] E. C. Fear, X. Li, S. C. Hagness and M. A. Stuchly, "Confocal Microwave Imaging for Breast Cancer Detection: Localization of Tumors in Three Dimensions," IEEE Transactions On Biomedical Engineering, vol. 49, no. 8, 2002.

[25] D. Byrne, M. O'Halloran, E. Jones, and M. Glavin, "Transmitter-grouping robust capon beamforming for breast cancer detection," Progress In Electromagnetics Research, Vol. 108, 401-416, 2010.

[26] W. Huang and A. A Kishk, "Compact wideband multi-layer cylindrical dielectric resonator antennas," IEE Proc. Microw. Antennas Propag., vol. 1, no. 4, pp. 998-1005, 2007.

[27] International Agency for Research on Cancer (IARC) and World Health Organization (WHO). GLOBOCAN 2012, Estimated cancer incidence, mortality and prevalence worldwide in 2012. 